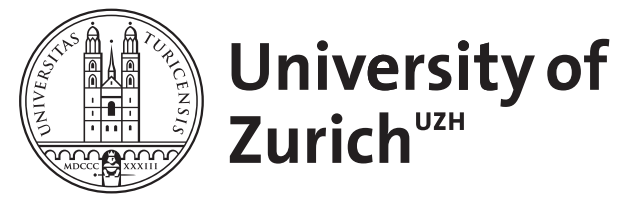

Zurich Open Repository and Archive

University of Zurich

University Library

Strickhofstrasse 39

CH-8057 Zurich

www.zora.uzh.ch

Year: 2015

\title{
Rechtsangleichung im Schweizer Finanzmarktrecht - Heteronomer und autonomer Nachvollzug, Äquivalenz, Swiss Finish
}

\author{
Sethe, Rolf
}

Posted at the Zurich Open Repository and Archive, University of Zurich

ZORA URL: https://doi.org/10.5167/uzh-116054

Book Section

Originally published at:

Sethe, Rolf (2015). Rechtsangleichung im Schweizer Finanzmarktrecht - Heteronomer und autonomer Nachvollzug, Äquivalenz, Swiss Finish. In: Kohte, Wolfhard; Absenger, Nadine. Menschenrechte und Solidarität im internationalen Diskurs: Festschrift für Armin Höland. Baden-Baden: Nomos, 345-365. 
Rechtsangleichung im Schweizer Finanzmarktrecht

- Heteronomer und autonomer Nachvollzug, Äquivalenz, Swiss Finish

Rolf Sethe

Rolf Sethe, Rechtsangleichung im Schweizer Finanzmarktrecht

- Heteronomer und autonomer Nachvollzug, Äquivalenz, Swiss Finish

in: Wolfhard Kohte/Nadinge Absenger (Hrsg.),

Menschenrechte und Solidarität im internationalen Diskurs,

Festschrift für Armin Höland,

Nomos-Verlag, Baden-Baden 2015

\section{Einleitung}

Ich habe Armin Höland als einen privat wie beruflich extrem vielseitigen und wissbegierigen Menschen kennengelernt. Aus dem breiten Spektrum seiner Interessen möchte ich an dieser Stelle nur zwei herausgreifen, die mich besonders beeindruckt haben, nämlich seine Neugier auf fremde Länder und Rechtsordnungen sowie sein Gefühl für eine gute Sprache.

Sein jugendlicher Charme öffnete ihm bei seinen zahlreichen Reisen viele Türen und er kann heute auf einen sehr reichhaltigen Erfahrungsschatz an Auslandskontakten zugreifen. Das, was viele Berufsratgeber neudeutsch als „Networking“ beschreiben und als bloßes Mittel zum Zweck begreifen, war für Armin Höland immer schon Herzensangelegenheit und gerade nicht zweckorientiert im Sinne eines beruflichen Vorankommens. Er sucht den Kontakt zu anderen Menschen ohne den Hintergedanken nach einem bestimmten Nutzen. Es war daher kein Wunder, dass die Juristische Fakultät der Martin-Luther-Universität Halle-Wittenberg ihn kurz nach seiner Berufung zum Außenbeauftragten, zum Erasmusbeauftragten und zum Leiter des Europäischen Dokumentationszentrums gewählt hat. Seine beiden Dekanate waren ebenfalls durch spannende Begegnungen mit Gästen aus aller Herren Länder gekennzeichnet.

Seine Vorliebe für Sprache entdeckt man in dem Moment, in dem man das erste Mal mit Armin Höland in Kontakt kommt. Ihm liegt Sprache am Herzen und er kann Dinge besser auf den Punkt bringen als jeder andere, den ich kenne. Dass dabei der Humor nicht zu kurz kommt, merkt man auch sehr schnell. Während seines ersten Dekanats hatte er die mühevolle

* Der vorliegende Beitrag ist im Rahmen des Universitären Forschungsschwerpunkts Finanzmarktregulierung entstanden. Der Verfasser dankt seinen Mitarbeitern lic. iur. Lukas Fahrländer und MLaw Fabio Andreotti für ihre wertvollen Anregungen. 
Aufgabe, sich um die Beseitigung eines Sachmangels am neu errichteten Juridicum in Halle zu kümmern. Dort ribbelte sich der Teppichboden der Bibliothek Schlaufe für Schlaufe auf. Armin Höland umschrieb dies in Anlehnung an die bekannte Rechtsfigur als einen ,sich selbst auffressenden Mangel“.

Sein Interesse an fremden Rechtsordnungen und an Sprache hat mich bewogen, ein Thema aus der Methodenlehre i.w.S. aufzugreifen, nämlich die Frage, inwieweit ein Schweizer Jurist bei Erlass und Auslegung von Normen ausländische Vorstellungen berücksichtigt oder gar berücksichtigen muss. Dabei wird das Finanzmarktrecht als Beispiel genommen. ${ }^{1}$

\section{Die Rechtsangleichung im Schweizer Finanzmarktrecht}

1. Wirtschaftliche Ausgangslage

Die Schweiz als relativ kleiner, rohstoffarmer Staat mit dennoch große Wirtschaftsleistung ist auf eine Vernetzung mit den im Weltmarkt wichtigen Akteuren angewiesen. Dies gilt in besonderem Maße für den Bereich der Finanzdienstleistungen, der 2011 insgesamt 10,3\% des Bruttoinlandsprodukts ausmachte. ${ }^{2}$

\begin{tabular}{|l|c|c|}
\hline Wertschöpfung des Finanzsektors (2011) & $\begin{array}{c}\text { Absolut } \\
\text { (in Mrd. CHF) }\end{array}$ & $\begin{array}{c}\text { Anteil am BIP } \\
\text { (in \%) }\end{array}$ \\
\hline Total Wertschöpfung Bankensektor & 35,0 & 6,2 \\
\hline davon: Retail Banking & 13,5 & 2,4 \\
\hline Wealth Management & 14,7 & 2,6 \\
\hline Asset Management & 4,1 & 0,7 \\
\hline Investment Banking & 2,7 & 0,5 \\
\hline Total Wertschöfung Versicherungssektor & 24,4 & 4,1 \\
\hline Total Wertschöpfung Finanzsektor & 59,4 & 10,3 \\
\hline
\end{tabular}

1 Eine umfassende Analyse in Bezug auf das Wirtschaftsrecht im Allgemeinen findet sich bei Heinemann, Rechtliche Transplantate zwischen Europäischer Union und der Schweiz, in: Fahrländer/Heizmann (Hrsg.), Europäisierung der schweizerischen Rechtsordnung, Zürich/St. Gallen 2013, 3 ff. Einen Überblick über das Finanzmarktrecht geben Weber/Schaller, Auswirkungen der EU-Finanzmarktregulierung marktrecht geben Weber/Schaller, Auswirkungen der EU-Finanzmarktregulierung auf die schweizerische Finanzmarktgesetzgebung, EuZ 2004, $74 \mathrm{ff}$

2 Swiss Banking, Der Finanzplatz Schweiz und seine Bedeutung, Juli 2012, http:// zuercher-bankenverband.ch/wp-content/uploads/FinanzplatzSchweizSBVg-2012.pdf
Banken und Finanzdienstleister sind zudem bedeutende Arbeitgeber in der Schweiz, da sie 5,7\% aller Beschäftigten stellen ${ }^{3}$

\begin{tabular}{|l|c|c|}
\hline Anzahl Beschäftigte (2011) & Finanzsektor & Bankensektor \\
\hline Beschäftigte im Inland & 195.834 & 142.800 \\
\hline Beschäftigte im Ausland & 180.269 & 106.760 \\
\hline Total Beschäftigte & 376.103 & 249.560 \\
\hline
\end{tabular}

Die Banken und sonstigen Finanzdienstleister (inkl. Mitarbeitende sowie Aktionäre) bezahlten 2011 zusammen jährlich schätzungsweise CHF 1418 Mrd. direkte und indirekte Steuern. Dies entspricht 12-15\% aller Steuereinnahmen von Bund, Kantonen und Gemeinden. ${ }^{4}$

Die Schweiz ist daher bestrebt, die rechtlichen Rahmenbedingungen für den Finanzsektor möglichst wettbewerbsfreundlich auszugestalten. Dabei lassen sich verschiedene Mechanismen beobachten, auf die nachfolgend eingegangen wird.

2. Mitwirkung an und Übernahme von internationalen Standards

Die Schweiz ist in zahlreichen Gremien aktiv, die sich mit der Entwicklung internationaler Standards befassen. Im Bereich des Finanzmarktrechts sind dies etwa das Basel Committee on Banking Supervision, der Financial Stability Board oder die International Organization of Securities Commissions (IOSCO). Um wettbewerbsfähig zu bleiben, übernimmt die Schweiz regelmäßig internationale Regulierungsstandards, wie etwa die Basler Eigenkapitalvorschriften, die Vorgaben zum Derivatehandel aufgrund der G20-Beschlüsse von Pittsburgh oder die Regelungen zur Bekämpfung der Geldwäscherei.

3. Übernahme von Rechtsregeln aufgrund wirtschaftlichen Drucks

Von dieser freiwilligen Übernahme, die auf einem konsensorientierten Verfahren zur Entwicklung neuer Rechtsregeln beruht, zu unterscheiden ist ein anderer in letzter Zeit vermehrt auftretender Mechanismus, nämlich

3 Vgl. Fn. 2.

4 Vgl. Fn. 2. 
die Übernahme von Rechtsregeln aufgrund des wirtschaftlichen Drucks eines (besonders wichtigen) Marktteilnehmers, was man als „heteronomen Nachvollzug“ bezeichnen könnte. Ein Beispiel für eine solche unfreiwillige Übernahme fremder Rechtsvorstellungen ist die faktische Abschaffung des Bankgeheimnisses gegenüber ausländischen Steuerbehörden aufgrun des Drucks, der vor allem von den USA, aber auch von Frankreich un Deutschland und der OECD ausging. ${ }^{5}$ Auch schon zuvor war die Schweiz mehrfach gezwungen, ihre Rechtsordnung an US-amerikanische Standards anzugleichen, um massive wirtschaftliche Nachteile zu vermeiden. Unter dem - zumeist negativ besetzten - Stichwort „lex americana“ werden im Finanzmarktrecht zwei Beispiele genannt: Einerseits schuf die Schweiz ein Insiderrecht, um Ersuchen der US-amerikanischen Justiz um Rechtshilfe genügen zu können. ${ }^{6}$ Andererseits war die Schweiz gezwungen, ein Revisionsaufsichtsgesetz ${ }^{7}$ einzuführen, als die USA nach den Skandalen um Enron und Worldcom den Sarbanes-Oxley-Act erließen, um bei börsennotierten Unternehmen Bilanzverstöße besser verhüten und ggf. strenger ahnden zu können; ${ }^{8}$ ansonsten hätten Schweizer Unternehmen, die in den USA börsennotiert sind, ihren Jahresabschluss doppelt (in den USA und der Schweiz) prüfen lassen müssen.

5 Das Beratungsunternehmen PwC schätzt, dass in den sechs Jahren 2008-2014 ca. 350 Mrd. CHF vom Schweizer Finanzplatz abgeflossen sind - vor allem von ausländischen Schwarzgeldkunden, NZZ v. 4.9.2014, 23 („,Die Weißgeldstrategie greift").

6 Art. 161 StGB wurde durch BG v. 18.12.1987 eingeführt und ist seit 1.7.1988 in Kraft, AS 1988, 966. Die Anfänge der Regulierung sind beschrieben bei Forstmoser, Effektenhandel durch Insider, SAG 1973, 133 ff.; Brunner, Wie kommt man den sogenannten Insidertransaktionen bei? SAG 1976, $179 \mathrm{ff}$. Forstmoser, Strafrechtliche Erfans rechtliche Erfassung von Insidermissbräuchen, SAG 1977, 14 ff.; Forstmoser, Wirt-
schaftsrecht im Wandel, SJZ 2008, 133, 134 f.

7 Bundesgesetz über die Zulassung und Beaufsichtigung der Revisorinnen und Revisoren (Revisionsaufsichtsgesetz, RAG) v. 16.12.2005, SR 221.302, AS 2007, 3971 Zu den Hintergründen für diese Regelung vgl. die Botschaft zu diesem Gesetz v. 23.6.2004, BBl 2004, 3969, 3979, $4004 \mathrm{ff}$.

8 Corporate and Auditing Accountability, Responsibility, and Transparency Act of 2002, (Sarbanes-Oxley Act), Pub. L. No. 107-204, 116 Stat. 745
4. Orientierung am europäischen Recht

\section{a) Grundsatz: Der Blick über die Grenze}

Das dritte und größte Einfallstor für fremde Rechtsregeln war und ist die Orientierung der Schweiz an den Regeln der EU. Die Schweiz ist nicht nur geographisch von EU- und EWR-Staaten umgeben, sondern diese sind auch die wichtigsten Handelspartner der Schweiz. Sie hat daher ein großes Interesse an Rahmenbedingungen, mit denen sie die Wettbewerbsfähigkeit ihrer Unternehmen auch in der EU stärkt.

Die Schweiz ist weder Mitglied der EU noch des EWR. Sie ist dahersieht man von einigen staatsvertraglichen Verpflichtungen einmal ab (dazu sogleich II.4.b) - nicht gehalten, das europäische Recht zu übernehmen. Dennoch schenkt sie den Entwicklungen im europäischen Unionsrecht eine große Beachtung. Dies kommt sehr deutlich in Art. 141 Abs. S. 2 lit. a ParlG ${ }^{9}$ zum Ausdruck. Nach dieser Vorschrift muss der Bundesrat der Bundesversammlung seine Gesetzentwürfe zusammen mit einer Botschaft unterbreiten, die eine Begründung des Gesetzes und - soweit nötig - der einzelnen Bestimmungen enthält. Dabei ist der Bundesrat verpflichtet, das Verhältnis zum europäischen Recht darzulegen. Diese Vorgaben werden im Gesetzgebungsleitfaden des EJPD ${ }^{10}$ und im Botschaftsleitfaden der Bundeskanzlei ${ }^{11}$ nochmals aufgegriffen. Indem man den Blick über die Grenze zu den unmittelbaren Nachbarn wirft, wird zum einen ein größerer Pool möglicher Lösungen für eine anstehende Ordnungsaufgabe aufgezeigt, zum anderen löst dieses Vorgehen faktisch einen Begründungszwang 12 aus, wenn man von der Lösung der EU abweichen will. Der Gesetzgebungsleitfaden drückt dies wie folgt aus: „Es geht bei diesem Streben nach Parallelität nicht darum, das europäische Rech automatisch nachzuvollziehen, wohl aber darum, zu verhindern, dass ungewollt und unnötigerweise neue Rechtsunterschiede geschaffen werden, welche die grundsätzlich angestrebte gegenseitige Anerkennung der Rechtsvorschriften auf europäischer Ebene behindern." Es darf allerdings

9 Bundesgesetz über die Bundesversammlung (Parlamentsgesetz, ParlG) v. 13.12.2002, SR 171.10, AS 2003, 3543

10 Gesetzgebungsleitfaden des Eidgenössischen Justiz- und Polizeidepartements, 3. Aufl., Bern 2007, Rn. $533 \mathrm{ff}$

1 Botschaftsleitfaden der Bundeskanzlei v. Juni 2013, Nr. 1.5.

12 Ebenso Heinemann (Fn. 1), 20 
nicht verkannt werden, dass die Übernahme des europäischen Rechts oftmals nicht aus Überzeugung erfolgt, sondern vor allem aus politischer Raison und wirtschaftlicher Notwendigkeit. ${ }^{13}$

Speziell im Bereich des Finanzmarktrechts finden sich weitere Vorgaben, die zum Blick über den Tellerrand nationaler Vorstellungen anhalten Art. 8 Abs. 3 des Bundesgesetzes über die Börsen und Effektenhandel (BEHG) verpflichtet die Börsen der Schweiz beim Erlass eines Reglements über die Zulassung von Effekten zum Handel dazu, internationa anerkannten Standards Rechnung zu tragen. Art. 7 Abs. 2 lit.b und d des Finanzmarktaufsichtsgesetzes (FINMAG), der die Regulierungsgrundsätze für die Eidgenössische Finanzmarktaufsicht FINMA enthält, verpflichtet die Behörde zu berücksichtigen, wie sich die Regulierung auf den Wettbewerb, die Innovationsfähigkeit und die internationale Wettbewerbsfähigkeit des Finanzplatzes Schweiz auswirkt und welche internationalen Mindeststandards gelten.

\section{b) Freiwillige Übernahme durch Staatsverträge}

Zwischen der EU und der Schweiz bestehen zahlreiche bilaterale Verträge. ${ }^{14}$ Grundlegend war und ist das Freihandelsabkommen von $1972,{ }^{15} \mathrm{mit}$ dem die Vertragsstaaten den freien Warenverkehr für Industrieprodukte einführten. Da das Volk und die Stände 1992 einen Beitritt zum EWR ablehnten, ${ }^{16}$ wird das Verhältnis der Schweiz zur EU seitdem durch sektorielle Verträge schrittweise ausgebaut, die zumeist eine Verpflichtung zur

13 Forstmoser, Der autonome Nach-, Mit- und Vorvollzug europäischen Rechts: das Beispiel der Anlagefondsgesetzgebung, in: FS Zäch, Zürich 1999, 523.

14 Ein Überblick findet sich auf der Seite des Eidgenössischen Departements für auswärtige Angelegenheiten (EDA) unter https://www.eda.admin.ch/content/dam/ed /de/documents/publications/EuropaeischeAngelegenheiten/FS-Bilaterale_de pdf Dazu Breitenmoser/Weyeneth, Europarecht, 2. Aufl., Zürich 2014, Rn. $632 \mathrm{ff}$; Felder/Kaddous, Accords bilatéraux Suisse - Union européenne (Comen2 r., Felder/Kaddons, Acor dilatéraux Suisse - Union européenne (Comentaires). B laterale Abkommen Schweiz - EU (Erste Analysen), Basel 2001; Jaag, Europarecht, 3. Aufl., Zurch 2010, Rn. 4101f., Kaddous/Jametti Greiner, Accords bilatéraux II Suisse-UE et autres Accords recents = Bilaterale Abkommen Schweiz-EU und andere neue Abkommen, Basel 2006; Thürer/Weber/Portmann Kellerhals, Bilaterale Verträge I und II Schweiz - EU, Zürich 2007.

15 Abkommen zwischen der Schweizerischen Eidgenossenschaft und der Europäischen Wirtschaftsgemeinschaft v. 22.7.1972, SR 0.632.401, AS 1972, 3115 .

16 http://www.bk.admin.ch/themen/pore/va/19921206/index.html?lang=de gegenseitigen Rechtsangleichung enthalten. Man versucht auf diese Weise, eine möglichst weitgehende Öffnung der Märkte zu erreichen. Mit dem Freizügigkeitsabkommen von $1999^{17}$ wurde ab 2002 die Arbeitnehmerfreizügigkeit und die Niederlassungsfreiheit für natürliche Personen eingeführt. Juristische Personen können nur Dienstleistungen im Umfang von 90 Tagen pro Jahr erbringen, eine dauerhafte Niederlassung ist ihnen verwehrt, es sei denn, sie fallen unter Spezialabkommen, wie z.B. das Versicherungsabkommen ${ }^{18}$ oder das Luftverkehrsabkommen. ${ }^{19}$ Auch die Dienstleistungsfreiheit ist bislang noch nicht allgemein gewährleistet. Vielmehr finden sich nur einzelne Regelungen in Spezialabkommen, wie etwa die erwähnte 90-Tage-Regel im Freizügigkeitsabkommen.

Im Bereich des Finanzmarktrechts finden sich keine Spezialabkommen. Das Freizügigkeitsabkommen sieht vielmehr eine Standstill-Klausel vor, wonach sich die mit dem Abkommen beabsichtigte Liberalisierung nicht auf Finanzdienstleistungen bezieht, für die im Hoheitsgebiet einer Vertragspartei eine vorherige Genehmigung erforderlich ist und deren Erbringer der Aufsicht der Behörden dieser Vertragspartei unterliegen (Art. 22 Abs. 3 ii des Anhangs I zum Freizügigkeitsabkommen). Da in der EU und in der Schweiz Banken und die meisten Finanzdienstleister seinerzeit bereits einer staatlichen Aufsicht unterworfen waren, gelten diese Vorschriften fort. Im Ergebnis besteht damit in Bezug auf den Finanzsektor keine Dienstleistungsfreiheit im bilateralen Verhältnis. ${ }^{20}$ Es bleibt folglich der Autonomie der EU bzw. des schweizerischen Gesetzgebers überlassen, welche Anforderungen an die Zulassung und den laufenden Betrieb von Bank- und Finanzdienstleistern gestellt werden.

17 Abkommen zwischen der Schweizerischen Eidgenossenschaft einerseits und der Europäischen Gemeinschaft und ihren Mitgliedstaaten andererseits über die Freizügigkeit v. 21.6.1999, SR 0.142.112.681, AS 2002, 1529.

18 Abkommen zwischen der Schweizerischen Eidgenossenschaft und der Europäischen Wirtschaftsgemeinschaft betreffend die Direktversicherung mit Ausnahme der Lebensversicherung v. 10.10.1989, SR 0.961.1, AS 1992, 1894.

19 Abkommen zwischen der Schweizerischen Eidgenossenschaft und der Europäischen Gemeinschaft über den Luftverkehr v 21.6.1999, SR 0.748.127.192.68, AS ischen Gemen. 1705 .

20 Das Memorandum zur Verbesserung der grenzüberschreitenden Tätigkeiten im Finanzbereich v. 3.7./15.8.2013, SR 0.672.913.631, AS 2013, 3677, regelt nur das Freistellungsverfahren für schweizerische Banken in der Bundesrepublik Deutschland. 
c) Freiwillige Übernahme im Wege des autonomen Nachvollzugs

aa) Gründe für den Nachvollzug im Allgemeinen

Die Schweiz hat in zahlreichen Gesetzen freiwillig rechtliche Vorgaben zum Bank- und Finanzdienstleistungsrecht aus der EU übernommen (sog. autonomer Nachvollzug'21), um die Wettbewerbsfähigkeit der Schweizer Banken und Finanzdienstleister zu fördern. ${ }^{22}$ Sie hatte sich durch einen Beitritt zum EWR und die damit einhergehende rechtliche Modernisierung (Übernahme des Acquis Communautaire durch das sog. „Eurolex“, mi dem 61 Gesetze geändert und neun neue verabschiedet werden sollten ${ }^{23}$ ) einen wirtschaftlichen Erneuerungsschub versprochen. Nach dem Scheitern des Beitritts im Jahr 1992 entschloss sich der Bundesrat, zahlreiche dieser Gesetzgebungsvorhaben dennoch umzusetzen, da man diese Modernisierungen des schweizerischen Rechts auch ohne Beitritt für notwendig erachtete. ${ }^{24} \mathrm{Im}$ Bereich des Finanzmarktrechts galt dies insbesondere für das Konsumentenkreditgesetz.

21 Unter einem autonomen Nachvollzug versteht man die nahezu unveränderte Übernahme europarechtlicher Regelungsmodelle. Davon zu unterscheiden sind Erlasse, bei denen das EU-Recht lediglich als "Quelle der Inspiration“ diente. Vgl. Heine mann (Fn. 1), 21, sowie Kohler, Le droit européen à l'aide de l'interprétation du droit suisse, in: Cottier (Hrsg.), Die Europakompatibilität des schweizerischen Wirtschaftsrechts: Konvergenz und Divergenz, Basel 2012, 41, 42 ff., die zwischen ,adaptation autonome stricto sensu“ und ,emprunt législatif“" unterscheidet.

22 Angesichts der Tatsache, dass mittlerweile bis zu 50\% des Bundesrechts durch EU-Recht beeinflusst sind (Oesch, Die Europäisierung des schweizerischen Rechts, in: Cottier (Hrsg.), Die Europakompatibilität des schweizerischen Wirtschaftsrechts: Konvergenz und Divergenz, Basel 2012, 13, 30; siehe auch Jenni, Direkte und indirekte Europäisierung der schweizerischen Bundesgesetzgebung, LeGes 2013, 489, die betont, dass sich der Einfluss der EU nicht in Prozenten messen lasse), sprechen manche auch vom Übergang vom ,autonomen zum systematischen Nachvollzug" Vgl. Spinner/Maritz. EG-Kompatibilität des schweistmatischen Nachollzug". Vgl. SpinnerManiz, EC-Konpatibilitat des schweizerschen Wirtschaftsrechts: Vom autonomen zum systematischen Nachvollzug, in

FS Zäch, Zuirch 199, 127fr., Zustimmen Heinemann (Fn. 1), 21

23 Botschaft I über die Anpassung des Bundesrechts an das EWR-Recht (Zusatzbotschaft I zur EWR-Botschaft) v. 27.5.1992, BBl 1992 V, 1; Botschaft II über die Anpassung des Bundesrechts an das EWR-Recht (Zusatzbotschaft II zur EWRBotschaft) v. 15.6.1992, BBl 1992 V, 520

24 Hierzu und zum Folgenden Botschaft über das Folgeprogramm nach der Ablehnung des EWR-Abkommens v. 24.2.1993, BBl $1993 \mathrm{I}, 805 \mathrm{ff}$
Ein weiterer Beleg für den autonomen Nachvollzug im Finanzmarktrecht ist die Reform des aus dem Jahre 1966 stammenden Schweizerischen Anlagenfondsgesetzes (AFG). ${ }^{25}$ Sie wurde durch die zunehmende Abwanderung dieses Geschäfts nach Luxemburg ausgelöst. ${ }^{26}$ Die 1992 in Angriff genommene und 1995 in Kraft getretene Revision des AFG verfolgte als Ziele die Wiederherstellung der Konkurrenzfähigkeit des Finanzplatzes Schweiz und die Steigerung seiner Attraktivität auf europäischer und internationaler Ebene, die Anpassung des Gesetzes an die europäische Regelung des Investmentrechts ${ }^{27}$ und die Verbesserung des Anlegerschutzes durch erhöhte Transparenz. ${ }^{28}$ Um dieses Ziel zu erreichen, schuf man mit der Reform u.a. EU-kompatible Anlagefonds und führte den in der damaligen EG bereits etablierten Prospektzwang sowie die Trennung von Fondsleitung und Depotbank ein. Diese Teile des AFG wurden also autonom nachvollzogen. Die Anpassung des schweizerischen Investmentrechts bewirkte allerdings nicht, dass die EU-kompatiblen Anlagefonds automatisch in der Union zugelassen waren, sondern führte nur dazu, dass sie die Anerkennungsvoraussetzungen erfüllten und daher überhaupt zum Vertrieb zugelassen werden konnten. ${ }^{29} \mathrm{Da}$ die OGAW-Richtlinie von $1985^{30}$ keine speziellen Vorgaben für Drittlandfonds enthielt, musste jeder Fonds einzeln den Antrag auf Anerkennung in dem jeweiligen Mitgliedstaat stellen. ${ }^{31}$

Im Jahre 2006 wurde das AFG durch das Kollektivanlagengesetz $(\mathrm{KAG})^{32}$ ersetzt. Auch hier belegen die Gesetzesmaterialien, wie stark diese Reform wiederum vom Gesichtspunkt der Wettbewerbsfähigkeit des Standorts Schweiz geprägt war, denn der Gesetzgeber verfolgte die Ziele

25 Bundesgesetz v. 1.7.1966 über die Anlagefonds (AFG), AS 1967, 115.

26 Botschaft zum revidierten Bundesgesetz über die Anlagefonds (Anlagefondsgesetz; AFG) v. 14.12.1992, BBl 1993 I, 217, 223 ff.; Forstmoser (Fn. 13), 524

27 RL 85/611/EWG v. 20.12.1985 zur Koordinierung der Rechts- und Verwaltungsvorschriften betreffend bestimmte Organismen für gemeinsame Anlagen in Wertv. 22.3.1988 Üb) die Änd L 3 ler RL 85/611/EWG, AB1. EG L 100 v. $19.4 .1988,31$

28 Botschaft AFG (Fn. 26), 218. Umfassend zu diesem Beispiel Forstmoser (Fn. 13), $523 \mathrm{ff}$.

29 Forstmoser (Fn. 13), $535 \mathrm{f}$

30 S.o. Fn. 27.

31 Botschaft AFG (Fn. 26), $223 \mathrm{f}$

32 Bundesgesetz über die kollektiven Kapitalanlagen (Kollektivanlagengesetz, KAG) v. 23.6.2006, SR 951.31, AS 2006, 5379. 
„der Wiederherstellung der Vereinbarkeit der schweizerischen Anlagefondsgesetzgebung mit der Regelung der Europäischen Union, den Ausbau der Anlagefondsgesetzgebung zu einer umfassenden Gesetzgebung über die kollektiven Kapitalanlagen, die Attraktivitätssteigerung sowie die Förderung der Wettbewerbsfähigkeit des schweizerischen Fondsplatzes [...] sowie eine differenzierte Ausgestaltung und ein maßvoller Ausbau des Anlegerschutzes durch zusätzliche Transparenz““.33 Man wollte also die zwischenzeitlich in der EU vorgenommenen Reformen im Bereich des Investmentrechts nachvollziehen. Hervorzuheben ist eine Regelung, die besonders deutlich zeigt, wie weit man bereit war zu gehen, um die EUKompatibilität zu erreichen: Investmentfonds war es nach EU-Recht erlaubt, die Anlageentscheidungen auf Vermögensverwalter zu delegieren, sofern diese einer staatlichen Aufsicht unterstanden. Da Vermögensverwalter in der Schweiz nicht beaufsichtigt werden, wäre es ihnen folglich nicht möglich gewesen, Fonds in der EU zu verwalten, wodurch ihnen ein Wettbewerbsnachteil entstanden wäre. Der Gesetzgeber sah daher vor, dass sich solche Vermögensverwalter freiwillig der Aufsicht durch die FINMA unterstellen konnten (Art. 13 Abs. 4 KAG a.F.)

Auch im Bereich des Börsenrechts vollzog man in vielen Bestimmungen das EU-Recht nach. Die Einführung des Börsengesetzes von 1994 war von dem Gedanken getragen, dass „mit einem modernen, den internationalen Standards entsprechenden Börsengesetz eine wichtige Voraussetzung für die Erhaltung der Wettbewerbsfähigkeit des Börsenplatzes Schweiz geschaffen [wird]“. ${ }^{34}$ Man orientierte sich - mit einigen Abweichungen im Detail - bei den Verhaltensregeln, bei der Offenlegung von Beteiligungen und bei Übernahmeangeboten inhaltlich an damals bereits in Kraft befindlichen oder als Entwurf vorliegenden Richtlinien. ${ }^{35}$ Auch die Börsenrechtsrevision von 2012, bei der die Reform des Verbots von Insiderhandel und Kursmanipulation im Mittelpunkt stand, erfolgte, um den internationalen Standards (GAFI-Empfehlungen und Übereinkommen des Euro-

33 Botschaft zum Bundesgesetz über die kollektiven Kapitalanlagen (Kollektivanlagengesetz) v. 23.9.2005, BBl 2005, 6395, 6396

34 Botschaft zu einem Bundesgesetz über die Börsen und den Effektenhandel (Börsengesetz, BEHG) v. 24.2.1993, BB1 1993, 1369, 1371

35 Botschaft (Fn. 34), 1387 ff., 1405 f.; a.A. Kunz, Amerikanisierung, Europäisierung sowie Internationalisierung im schweizerischen (Wirtschafts-)Recht, recht 2012, 37, 48 Fn. 187, der den Hinweis in der Botschaft am Ende von 5.15 missversteht: Die Botschaft nimmt nur die in den Abschnitten 5.11-5.15 genannten Richtlinien aus, nicht aber auch die übrigen. parats vom 16.5.2005 über Geldwäscherei und Terrorismusfinanzierung) zu genügen, aber auch um eine Lücke im Vergleich zum europäischen Recht zu schließen. ${ }^{36}$

bb) Nachvollzug zur Erreichung des Marktzugangs

Die Zahl der Beispiele ließe sich weiter fortsetzen. Für die vorliegende Untersuchung wichtig ist jedoch ein Blick auf die mit dem autonomen Nachvollzug verfolgten Ziele. Bei den Beispielen der Reform des Konsumentenkreditgesetzes von 1993 oder der Börsenrechtsrevision von 2012 ging es jeweils um die Modernisierung des inländischen Marktes, für die man das EU-Recht als Vorbild nahm. Betrachtet man dagegen die Reform des AFG, verband die Schweiz mit der einseitigen Änderung ihres Rechts die Erwartung auf einen erleichterten Zugang ihrer Finanzdienstleister zur Union. Wie bei allen bilateralen Vorgängen bedurfte der Marktzugang jedoch auch der Mitwirkung der anderen Seite. Der autonome Nachvollzug von europarechtlichen Vorgaben durch die Schweiz zum Zwecke des erleichterten Marktzugangs war also eine notwendige, aber keine hinreichende Bedingung für den Marktzugang.

Die Vorgaben der EU bzw. ihrer Mitgliedstaaten für die Gewährung des Marktzugangs haben sich im Laufe der Jahre sehr deutlich gewandelt. Betrachtet man die ersten von der Schweiz nachvollzogenen EU-Richtlinien, enthielten diese noch keine Regelung für den Zugang von Banken und Finanzdienstleistern aus Drittstaaten. So machte die erwähnte OGAW-IRichtlinie keine Vorgaben für die Zulassung von Anbietern aus Drittstaaten in der EU. Gleiches gilt für die Wertpapierdienstleistungsrichtlinie von $1993^{37}$ oder die sie ersetzende Richtlinie über Märkte für Finanzinstrumente von 2004 (nach ihrem englischen Akronym im Folgenden MiFID $\left.\mathrm{I}^{38}\right)$, die das Anbieten von Geschäften in Finanzinstrumenten regelt. Somit konnten die EU-Mitgliedstaaten autonom entscheiden, ab welcher Schwelle eine vom Drittstaat aus erbrachte Finanzdienstleistung in die EU hinein

36 Botschaft zur Änderung des Börsengesetzes (Börsendelikte und Marktmissbrauch) v. 31.8.2011, BB1 2011, 6873, 6874 .

37 RL 93/22/EWG v. 10.5.1993 über Wertpapierdienstleistungen, ABl. EG L 14 v. 11.6.1993, 27.

38 RL 2004/39/EG v. 21.4.2004 über Märkte für Finanzinstrumente, ABl. EU L 145 v. $30.4 .2004,1$ 
eine Bewilligungspflicht für den Anbieter auslöste und zu welchen Konditionen man den Finanzdienstleister anerkannte. Deutschland etwa ging bis 2004 davon aus, dass ein Anbieten im Inland nur vorliegt, wenn eine physische Präsenz im Inland besteht. Seit 2005 folgt man nun dem Auswirkungsprinzip (Marktortprinzip), so dass die Bewilligungspflicht bereits ausgelöst wird, wenn sich der Anbieter aus dem Drittstaat zielgerichtet an den inländischen Markt wendet. ${ }^{39}$ Ist dies der Fall, muss das aus einem Drittstaat stammende Institut in Deutschland eine Lizenz als Bank oder Finanzdienstleister nach $\S 32$ i.V.m. $\$ 1$ Abs. 1 und Abs. 1a KWG beantragen und damit die Vorgaben des deutschen Aufsichtsrechts einhalten.

Die EU hat in jüngerer Zeit gleich in mehreren Rechtsakten zum Finanzmarktrecht (z.B. Art. 34 ff. der AIFM-Richtlinie ${ }^{40}$, Art. 35, 75 EMIR $^{41}$, Art. 46 ff. MiFIR ${ }^{42}$, Art. 39 ff. MiFID II ${ }^{43}$ ) ihr Konzept, wonach die Frage des Zugangs von Unternehmen aus Drittstaaten zum Binnenmarkt ganz den Mitgliedstaaten überlassen wird, aufgegeben und macht nun ihrerseits vereinheitlichende Vorgaben in Verordnungen oder gibt den Mitgliedstaaten solche in Richtlinien vor. Dieser Paradigmenwechsel, der aus Platzgründen nicht vertieft werden kann, ${ }^{44}$ hat verschiedene Ursachen. Zum einen ist die EU an einer weiteren Marktöffnung für Produkte und/ oder Anbieter von Finanzdienstleistungen aus Drittstaaten interessiert, ${ }^{45}$

39 BaFin, Merkblatt - Hinweise zur Erlaubnispflicht nach $\S 32$ Abs. 1 KWG in Verbindung mit $\S 1$ Abs. 1 und Abs. 1a KWG von grenzüberschreitend betriebenen Bankgeschäften und/oder grenzüberschreitend erbrachten Finanzdienstleistungen v. 1.4.2005. Ausführlich dazu Sethe, in: Schäfer/Sethe/Lang (Hrsg.), Handbuch der Vermögensverwaltung, München 2012, § 4 N $108 \mathrm{f}$.

40 RL 2011/61/EU v. 8.6.2011 über die Verwalter alternativer Investmentfonds un zur Änderung der RL 2003/41/EG und 2009/65/EG und der Verordnungen (EG) 1060/2009 und (EU) 1095/2010, ABl. EU L 174 v. 1.7.2011, 1 .

41 Verordnung (EU) 648/2012 v. 4.7.2012 über OTC-Derivate, zentrale Gegenparteien und Transaktionsregister, ABl. EU L 201 v. 27.7.2012, 1.

42 Verordnung (EU) 600/2014 v. 15.5.2014 über Märkte für Finanzinstrumente un zur Änderung der Verordnung (EU) 648/2012, ABl. EU L 173 v. 12.6.2014, 84 .

43 RL 2014/65/EU v. 15.5.2014 über Märte für Finanzinstrumente sowie ü Ände RL 2014/65/EU Ver rung der RL $12.6 .2014,349$.

44 Ausführlich Zetzsche, Drittstaaten im Europäischen Bank- und Finanzmarktrecht in: Bachmann/Breig (Hrsg.), Finanzmarktregulierung zwischen Innovation un Kontinuität in Deutschland, Europa und Russland, Tübingen 2014, $47 \mathrm{ff}$.

45 Den Wunsch der EU nach Einbeziehung der Drittstaaten brachte bereits Erwägungsgrund 29 der Wertpapierdienstleistungsrichtlinie deutlich zum Ausdruck: „Da die Gemeinschaft ihre Kapitalmärkte für die anderen Länder offenhalten will, denn sie bewirkt eine Markterweiterung und schafft mehr Liquidität, Innovation sowie Produktdifferenzierung. Zudem sorgt sie für mehr Wettbewerb. Diese Öffnung birgt jedoch auch Gefahren, wie etwa Externalitäten für den Binnenmarkt, wenn Anbieter aus Drittstaaten weniger streng beaufsichtigt werden als Anbieter aus der EU (contagion, spill over-Effekte). Zudem will man die eigenen Marktteilnehmer vor unseriösen Angeboten aus dem Ausland schützen und eine Regulierungsarbitrage bzw. ein race to the bottom im Aufsichtsrecht verhindern. ${ }^{46}$ Ansonsten würde eine Wettbewerbsverzerrung zulasten der heimischen Anbieter ausgelöst, wenn Drittstaaten ihr Aufsichtsrecht laxer gestalten (dürfen) als die EU. Sowohl die EU als auch ihre Mitgliedstaaten haben daher ein Interesse an der Regulierung des Zugangs von Produkten oder Anbietern aus Drittstaaten zur EU. Aus Sicht der Schweiz ist dabei vor allem die Frage wichtig, ob die Anforderungen für schweizerische Banken und Finanzdienstleister von der EU oder von den einzelnen Mitgliedstaaten bestimmt werden. Eine Zentralisierung der Anforderungen hat den Vorteil, dass man nur einen, d.h. einheitlichen Standard zu beachten hat.

Das neue Konzept sieht jeweils vor, dass Anbieter aus Drittstaaten nur Zugang zum EU-Markt erhalten, wenn diese in ihrem Heimatland einer effektiven und gleichwertigen Aufsicht unterliegen. Darüber hinaus werden in manchen Rechtsakten auch inhaltliche Anforderungen an das Aufsichtsrecht des Drittstaates gemacht, das demjenigen der EU äquivalent sein muss. Beispielhaft ${ }^{47}$ genannt sei die Regelung der VO (EG) 600/2014 über Märkte für Finanzinstrumente (MiFIR) für das Erbringen von grenzüberschreitenden Wertpapierdienstleistungen gegenüber geeigneten Gegenparteien und geborenen professionellen Kunden (Art. 46 Abs. 2 lit. a, Art. 47 Abs. 1 MiFIR). Danach ist erforderlich, dass die EU-Kommission einen Beschluss fasst, ob sie die Finanzmarktregulierung des Drittstaates als aufsichtsrechtlich äquivalent erachtet. Der Drittstaat hat keinen Anspruch auf einen solchen Beschluss, geschweige denn auf ein Eintreten in diese Sachprüfung. Die Kriterien der Gleichwertigkeitsprüfung sind in Art. 47 Abs. 1

ist das Ziel dieses Verfahrens nicht eine Abschottung der Kapitalmärkte der Gemeinschaft, sondern eine weitergehende Liberalisierung der gesamten Kapitalmärkte in den Drittländern."

46 Ebenso Eggen/Schaefer, Regulierung grenzüberschreitender Tätigkeiten im Finanzmarktrecht, GesKR 2013, 368.

47 Ausführlich dazu Sethe, Das Drittstaatenregime von MiFIR und MiFID II, SZW $2014,615 \mathrm{ff}$ 
MiFIR niedergelegt. Zu betonen ist, dass Äquivalenz gerade nicht Deckungsgleichheit bedeutet, sondern Vergleichbarkeit. Es müssen also alle wesentlichen Elemente des Aufsichtsrechts (Kernprinzipien) übernommen werden. ${ }^{48}$ Dies stellt die MiFIR ausdrücklich klar, wenn sie verlangt, dass die Gleichwertigkeitsprüfung ,,ergebnisorientiert“" sein muss. ${ }^{49}$

Um die Äquivalenz mit MiFID II/MiFIR zu erreichen, hat die Schweiz zwei Gesetzgebungsvorhaben auf den Weg gebracht, deren Vernehmlassung ${ }^{50}$ soeben abgeschlossen wurde..$^{51}$ Das geplante Finanzinstitutsgesetz (FINIG) wird künftig nicht nur Banken und Effektenhändler einer Zulassungspflicht und einer prudentiellen Aufsicht unterwerfen, sondern auch Vermögensverwalter (Art. 17 ff. VE-FINIG). Hingegen sollen Anlageberater weiterhin nicht umfassend prudentiell beaufsichtigt werden. Sie bedürfen nach Art. 30 des geplanten Finanzdienstleistungsgesetzes (FIDLEG) keiner Zulassung, wohl aber einer Registrierung. Dazu müssen sie ihre Zuverlässigkeit und fachliche Eignung sowie eine Haftpflichtversicherung und den Anschluss an eine Ombudsstelle nachweisen (Art. 28-30 VEFIDLEG). Auch die Wohlverhaltensregeln und Organisationsanforderungen der MiFID II sollen weitgehend übernommen werden (Art. $1 \mathrm{ff}$. VEFIDLEG).

d) Zwischenfazit

Mit dem Konzept der Äquivalenz exportiert die EU letztlich ihre Regulierungsstandards in Drittstaaten. Diese werden nicht zwei Regelungsregime vorhalten (eines für rein inländisch orientierte Institute und eines für Institute, die den Zugang zur EU wollen), sondern werden der Einfachheit halber ihr Finanzmarktrecht der jeweiligen EU-Regelung anpassen (was of auch unter Gesichtspunkten wie Anlegerschutz oder Konformität mit Marktstandards der bessere Wege ist). Dabei ist der Umfang notwendiger

48 Ebenso Zetzsche (Fn. 44), 128; Weber/Sethe, Äquivalenz als Regelungskriterium im Finanzmarktrecht, SJZ 2014, 569, 575

49 Erwägungsgrund 41 Unterabs. 3 Satz 1 MiFIR. Zu den beiden Maßstäben der „wörtlichen“ und der ,ergebnisorientierten“ Äquivalenz auch Eggen/Schaefer (Fn. 46), 373.

50 Zu diesem Verfahren Karpen, Schweizerische Rechtsetzung und Rechtsetzungslehre - ein Blick von außen, ZG 2012, $68 \mathrm{ff}$.

$51 \mathrm{http}: / / \mathrm{www} \cdot$ admin.ch/aktuell/00089/index.html?lang=de\&msg-id=53561 (zuletz abgerufen am 22.1.2015)
Reformen im Einzelfall sehr unterschiedlich. Handelt es sich um einen Bereich des Finanzmarktrechts, der schon durch internationale Standards (Basel Committee, IOSCO, FSB etc.) faktisch harmonisiert wurde, wird bereits deshalb Äquivalenz bestehen; 52 andernfalls sind weitreichendere Eingriffe in das Recht des Drittstaats nötig, wie jetzt das Beispiel des Nachvollzugs von MiFID II/MiFIR in der Schweiz zeigt.

\section{Folgen für die Methodenlehre}

Interessant ist es zu beobachten, dass der schweizerische Gesetzgeber zahlreiche ausländische Regelungen nicht 1:1 übernahm, sondern oftmals Sonderregelungen zugunsten der heimischen Anbieter vorsah. Dieser im anglo-amerikanischen als „Cherry-Picking“ bekannte Vorgang trägt in der Schweiz den Namen „Swiss Finish“. Da der schweizerische Gesetzgeber die ausländische Regelung freiwillig zum Vorbild nimmt, bleibt es ihm unbenommen, diese nur unvollständig zu übernehmen ${ }^{53}$ oder über sie hinauszugehen. ${ }^{54}$ Das „Swiss Finish“ ist zu unterscheiden von einem ähnlichen Phänomen, das man in der EU kennt, nämlich das sog. „Gold Plating". Gemeint ist damit eine Richtlinienumsetzung, bei der der Mitgliedstaat bewusst über die in der Richtlinie vorgegebene Mindestharmonisierung hinausgeht. „Gold Plating“ stellt also regelmäßig eine Verschärfung des nationalen Rechts im Vergleich zur Richtlinie dar und benachteiligt typischerweise die heimischen Anbieter im Vergleich zu Anbietern aus

52 Hierauf weist Zetzsche (Fn. 44), 127, hin.

53 Beispielsweise hat der schweizerische Gesetzgeber nur die Wohlverhaltensregeln aus der Wertpapierdienstleistungsrichtlinie 1993 übernommen, Sethe, Treuepflichten der Banken bei der Vermögensanlage, AcP 2012, 80, 117, nicht aber auch die Aufsicht über unabhängige Vermögensverwalter.

54 Ein Beispiel hierfür findet sich in der erwähnten Revision des AFG von 1995: Art. 9 Abs. 6 AFG schrieb nicht nur die von der Richtlinie verlangte Trennung von Fondsleitung und Depotbank vor, sondern auch eine personelle Trennung auf der Sondsleitung und Depotbank vor, sondern auch eine personelle Trennung auf der

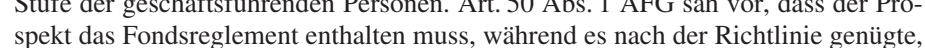
dass dieses Dokument auf Wunsch bezogen werden konnte, vgl. Forstmoser $(\mathrm{Fn}$ 13), 530. Ein weiteres Beispiel sind die gegenüber Basel II und Basel III erhöhten Eigenkapitalanforderungen an schweizerische Banken, vgl. etwa Staub, Da schweizerische Bankenaufsichtsrecht und die Kreditkrise, GesKR 2009, 499, 508. 
anderen EU-Staaten. ${ }^{55}$ Vor dem Hintergrund der soeben beschriebenen Entwicklung hin zu einer Äquivalenzprüfung ist allerdings der Spielraum, der für ein „Swiss Finish“ verbleibt, u.U. sehr viel kleiner als früher.

Die freiwillige Übernahme fremden Rechts hat natülich auch Auswirkungen auf die Methoden zur Auslegung dieses Rechts. Nach Ansicht des Bundesgerichts ist es insbesondere im traditionell grenzüberschreitenden Rechtsverkehr geboten, bei der richterlichen Auslegung und Lückenfüllung (Art. 1 Abs. 2 ZGB) eine rechtsvergleichende Betrachtung vorzunehmen, um einen isolationistischen Rechtszustand zu vermeiden, der entweder durch Privilegierungen oder durch Diskriminierungen auf dem internationalen Markt ausgelöst werden könne. ${ }^{56}$ Über diese für sämtliche grenzüberschreitenden Fälle geltende Vorgabe geht das Bundesgericht noch hinaus, wenn es bei Vorschriften, die im Wege des autonomen Nachvollzugs von EU-Richtlinien ins Schweizer Recht transplantiert wurden, eine europarechtskonforme Auslegung vorgibt: „Das Recht der Europäischen Union entfaltet zwar keine unmittelbaren verbindlichen Auswirkungen auf das schweizerische Recht. Da die Revision 1993 die Anpassung von Art. 333 OR an die Richtlinie 77/187 des EWG-Rates vom 14. Februar 1977 im Rahmen des so genannten autonomen Nachvollzugs des europäischen Rechts bezweckte [...], ist die EU-Rechtsordnung indessen als Auslegungshilfe beizuziehen [...]. Nachvollzogenes Binnenrecht ist im Zweifel europarechtskonform auszulegen. Es ist harmonisiertes Recht und als solches im Ergebnis - wie das Staatsvertragsrecht - Einheitsrecht. Zwar ist es nicht Einheitsrecht in Form von vereinheitlichtem Recht. Wird aber die schweizerische Ordnung einer ausländischen - hier der europäischen - angeglichen, ist die Harmonisierung nicht nur in der Rechtssetzung, sondern namentlich auch in der Auslegung und Anwendung des Rechts anzustreben, soweit die binnenstaatlich zu beachtende Methodologie eine solche Angleichung zulässt." 57 In der Sache ist dies konsequent, denn die mit der Übernahme des EU-Rechts bezweckte Angleichung würde faktisch wieder ausgehebelt, wenn die nachfolgende Rechtsanwendung

55 So wird etwa das Finanzkommissionsgeschäft von der MiFID I als Finanzdienstleistung begriffen, Deutschland hat es aber strenger reguliert, indem es in $\$ 1$ Abs. 1 Satz 2 Nr. 4 KWG als Bankgeschäft eingeordnet ist.

56 BGE 126 III 129, 138 E. 4.

57 BGE 129 III 335, 350 E. 6; ebenso BGE 125 II 293, 306 f. E. 4e; BGE 130 III 182 190 E. 5.5.1; BGE 133 III 180, 184 E. 3.5; BGE 137 III 487, 495 E. 4.6; BGer v. 30.4.2004, 2A.334/2003 E. 2.3; zustimmend etwa Kunz (Fn. 35), 49 f. sich vom „Original“ entfernt. Für die Praxis besteht die Schwierigkeit, wann genau die europarechtskonforme Auslegung zur Anwendung kommt. Die Lehre verlangt, dass die fragliche Bestimmung im Wege des autonomen Nachvollzugs geschaffen wurde und nicht lediglich europarechtlich inspiriert ist. Ausschlagend dafür ist der in den Gesetzesmaterialien zum Ausdruck kommende Wille des schweizerischen Gesetzgebers, ${ }^{58}$ wobei die Grenzziehung im Einzelfall schwierig sein kann. Hierau kommt es jedoch letztlich nicht an, denn auch wenn es sich nicht um einen autonomen Nachvollzug im technischen Sinne handelt, ist das Bundesgericht nicht gehindert, im Rahmen der allgemeinen Auslegung ausländische Rechtsvorstellungen beizuziehen. ${ }^{59}$ Ist im konkreten Fall die europarechtskonforme Auslegung anzuwenden, hat dies zwei Auswirkungen: (1) Das Bundesgericht verlangt, dass die europarechtskonforme Auslegung nur im Rahmen der binnenstaatlich zu beachtenden Methodologie anzuwenden ist. Seiner Ansicht nach kommt der europarechtskonformen Auslegung somit keine eigenständige Rolle neben den übrigen Auslegungselementen zu. $^{60}$ (2) Weiterhin sind sämtliche Quellen des entsprechenden EU-Rechts zu konsultieren, so dass nicht nur die umgesetzte Richtlinie, sondern auch eine nachfolgende EuGH-Rechtsprechung maßgebend ist. ${ }^{61}$

58 Cottier/Dzamko/Evtimov, Die europakompatible Auslegung des schweizerische Rechts, Schweizerisches Jahrbuch für Europarecht 2003/Annuaire suisse de Droit européen 2003, Bern 2004, 364; Kunz, Europa als ein Maßstab für das schweizerische Wirtschaftsrecht?, FS Bucher, Bern 2009, 455, 465; Beusch, Der Einfluss „fremder" Richter - Schweizer Verwaltungsrechtspflege im internationalen Kontext, SJZ 2013, 349, 356; Jaag (Fn. 14), Rn. 4206; ferner Amstutz, Interpretatio multiplex, in: FS Kramer, Basel 2004, 86 ff. m.w.N.; Walter, Das rechtsvergleichende Element - Zur Auslegung vereinheitlichten, harmonisierten und rezipierten chende Element - Zur Auslegu

59 BGE 126 III 129, 138 E. 4; Walter (Fn. 58), 259 ff.; Schluep, Einladung zur Rechtstheorie, Bern 2006, Rn. 1620 ff.; Amstutz (Fn. 58), 67 ff.; Jaag (Fn. 14), Rn. 4207a; a.A. Kunz, Umgang mit internationalem und mit europäischem Recht. Überblick über den „,Swiss Approach“, LeGes 2012/3, 265, 270.

60 Zu Recht kritisch Amstutz (Fn. 58), 90 f.; Wiegand/Brülhart, Die Auslegung von autonom nachvollzogenem Recht der Europäischen Gemeinschaft, Bern 1999, 38 a.A. $\operatorname{Kunz}($ Fn. 59$), 270$

61 Ebenso Kunz (Fn. 58), 465. 


\section{Sprachlicher Ausdruck}

Angesichts von Armin Hölands Vorliebe für gute Sprache, liegt es nahe, nicht nur den Weg von „legal transplants“ ins Schweizer Recht zu betrachten, sondern auch das sprachliche Ergebnis. Die Sprache des Schweizer Gesetzgebers ist einfach, klar und verständlich. Sie unterscheidet sich damit grundlegend von der ,verquasten“62 Amtssprache der EU, die nicht zuletzt durch den Zwang zu Kompromissen unter den 28 Mitgliedstaaten und die zahlreichen Übersetzungsprobleme aufgebläht wird. Sie unterscheidet sich aber auch vom Sprachgebrauch des deutschen Gesetzgebers, der keinen solchen Sachzwängen ausgesetzt ist wie die EU. Dies wird am Beispiel des Art. 19 Abs. 5 der MiFID I und seiner Umsetzung in Deutschland sowie der Schweiz verdeutlicht. Die Vorschrift regelt den sog. Angemessenheitstest. Danach ist ein Finanzintermediär verpflichtet, seinen Wertpapierkunden zu warnen, wenn dieser Geschäfte ohne vorherige Anlageberatung in Auftrag gibt und diese erkennbar für seine Verhältnisse unangemessen sind. Der identische Inhalt ist in den drei Rechtsordnungen wie folgt formuliert:

\begin{tabular}{|c|c|c|}
\hline EU: Art. 19 Abs. 5 MiFID I & D: $\$ 31$ Abs. 5 WpHG & CH: Art. 11 VE-FIDLEG \\
\hline $\begin{array}{l}\text { „Die Mitgliedstaaten stellen si- } \\
\text { cher, dass Wertpapierfirmen bei } \\
\text { anderen als den in Absatz } 4 \text { ge- } \\
\text { nannten Finanzdienstleistungen } \\
\text { von Kunden oder potenziellen } \\
\text { Kunden Angaben zu ihren } \\
\text { Kenntnissen und Erfahrungen im } \\
\text { Anlagebereich in Bezug auf den } \\
\text { speziellen Typ der angebotenen } \\
\text { oder angeforderten Produkte } \\
\text { oder Dienstleistungen einholen, } \\
\text { um beurteilen zu können, ob die } \\
\text { in Betracht gezogenen Wertpa- } \\
\text { pierdienstleistungen oder Pro- } \\
\text { dukte für den Kunden geeignet } \\
\text { sind." }\end{array}$ & \begin{tabular}{|l}
, Vor der Erbringung anderer \\
als der in Absatz 4 genannten \\
Wertpapierdienstleistungen zur \\
Ausfuhtunung von Kundenaufträ- \\
gen hat ein Wertpapierdienst- \\
leistungsunternehmen von den \\
Kunden Informationen über \\
Kennntissse und Erfahrungen \\
der Kunden in Bezug auf Ge- \\
schäfte mit bestimmten Arten \\
von Finanzinstrumenten oder \\
Wertpapierdienstleistungen \\
einzuholen, soweit diese Infor- \\
mationen erforderlich sind, um \\
die Angemessenheit der Fi- \\
nanzinstrumente oder Wertpa- \\
pierdienstleistungen für die \\
Kunden beurteilen zu können."
\end{tabular} & $\begin{array}{l}\text { "Ein Finanzdienstleister, der an- } \\
\text { dere Finazzdienstleistungen als } \\
\text { solche nach Artikel } 10 \text { erbringt, } \\
\text { erkundigt sich über die Kennt- } \\
\text { nisse und Erfahrungen seiner } \\
\text { Kundinnen und Kunden in Be- } \\
\text { zug auf die angebotenen Produk- } \\
\text { te oder Dienstleistungen und } \\
\text { prüft vor der Dienstleistungser- } \\
\text { bringung, ob diese für die Kun- } \\
\text { dinnen und Kunden angemessen } \\
\text { sind.“ }\end{array}$ \\
\hline
\end{tabular}

Man sieht, wie erfrischend kurz und verständlich der autonome Nachvollzug im geplanten Schweizer FIDLEG geraten ist. Ein ähnliches Bild zeigt

62 Sehr anschaulich dazu Schröder, Europa in der Finanzfalle - Irrwege internationaler Regulierung, Berlin 2012, $75 \mathrm{ff}$. sich, wenn man die Bezeichnungen und Abkürzungen von Gesetzen und Verordnungen betrachtet. Die EU benötigt zumeist mehrere Zeilen, um eine Richtlinie zu bezeichnen. In Deutschland werden die Namen für Gesetze im Rahmen der sog. Rechtsförmlichkeitsprüfung vergeben, die nur ein Ziel hat, nämlich die eine Bezeichnung zu finden, die möglichst präzise den Inhalt des Gesetzes bzw. der Verordnung wiedergibt. Auf die sprachliche Qualität kommt es leider nicht an. Es finden sich Beispiele wie „Verordnung zur Konkretisierung der Verhaltensregeln und Organisationsanforderungen für Wertpapierdienstleistungsunternehmen“. Schon dieses Ungetüm lässt sich nur schwer aussprechen. Bei der Abkürzung „WpDVerOV“ ist dies schlicht ein Ding der Unmöglichkeit. Man gewinnt bisweilen den Eindruck, als hätten sich die mit der Rechtsförmlichkeitsprüfung beschäftigten Personen von der Realität emanzipiert. Dies musste Armin Höland als Dekan selbst erfahren, denn es ist ihm seinerzeit nich gelungen, beim Ministerium zu erreichen, dass die Verordnung über die Juristenausbildung ${ }^{63}$ eine Abkürzung erhält, die man flüssig aussprechen konnte. JAPrVO will einem einfach nicht über die Lippen gehen. Pragmatik kommt nicht vor in der deutschen Gesetzgebungsmaschinerie. ${ }^{64}$

V. Fazit

1. Die Rechtsangleichung im Schweizer Finanzmarktrecht erfolgt teils aufgrund wirtschaftlichen Drucks aus dem Ausland (heteronomer Nachvollzug), teils aufgrund staatsvertraglicher Verpflichtungen und teils aus eigener Entscheidung (autonomer Nachvollzug). Der autonome Nachvollzug ist aufgrund der Marktgegebenheiten oft nicht wirklich autonom im Sinne von freiwillig, sondern erweist sich als notwendig, um Standortvorteile zu erhalten. An dieser Stelle zeigt sich, welch

63 Ausbildungs- und Prüfungsverordnung für Juristen (JAPrVO) v. 2.10.2003, GVBl. LSA 2003, 245.

64 Im Handbuch der Rechtsförmlichkeit, hrsg. vom Bundesministerium der Justiz, 3. Aufl., Berlin 2008, http://hdr.bmj.de/vorwort.html, findet sich das Stichwort „Pragmatik“ nicht; anders die Weisungen der Schweizer Bundeskanzlei zur Schreibung und zu Formulierungen in den deutschsprachigen amtlichen Texten des Bundes, 2. Aufl., Bern 2013, Rn. 105: „Gehen Sie dort, wo die Schreibweisungen keine verbindlichen Regeln formulieren, pragmatisch vor: Suchen Sie die Lösung, die sich in der konkreten Verwendungssituation am besten eignet." 
geringer Gestaltungsspielraum dem nationalen Gesetzgeber im Finanzmarktrecht aufgrund der starken Verflechtung der Märkte verbleibt.

2. Unter dem Stichwort „autonomer Nachvollzug“ wird in der Schweiz der Vorgang verstanden, EU-Recht nachzubilden. Im Finanzmarktrecht erfolgt ein solcher Nachvollzug zum einen, um das inländische Rech an moderne Entwicklungen anzupassen; das EU-Recht dient in diesem Fall lediglich als unverbindliches Vorbild. Zum anderen aber finden sich auch zahlreiche Beispiele, in denen der Nachvollzug das Ziel verfolgt, den heimischen Banken und Finanzdienstleistern den Zugang zum EU-Binnenmarkt zu erleichtern. Das EU-Recht erfüllt in diesem Fall nicht nur die Funktion eines Vorbilds, von dem man auch abweichen kann, sondern es muss zu einem großen Teil inhaltlich deckungsgleich nachvollzogen werden, um die Voraussetzung des Marktzugangs, nämlich Äquivalenz mit der einschlägigen EU-Regelung, zu erreichen.

3. Die Übergänge zwischen der freiwilligen Übernahme von fremdem Recht zur Verbesserung der Wettbewerbsfähigkeit einerseits und de Übernahme aufgrund eines wirtschaftlichen Drucks andererseits sin also fließend, denn die Verbesserung der Wettbewerbsfähigkeit stellt einen faktischen Zwang dar. Der wesentliche Unterschied besteht in der Frage, von wem die Initiative zur Rechtsangleichung ausgeht. Bildlich gesprochen lassen sich „innerer Druck“ und „äußerer Druck“ unterscheiden.

4. Der Zugang zum Europäischen Finanzmarkt ist nicht einseitig zu erreichen. Vielmehr geben die Mitgliedstaaten bzw. die EU die Rahmenbedingungen vor, unter denen der Zugang gewährt wird. Die Schweiz wird daher - ohne dass sie eine inhaltliche Mitsprache bei der Entwicklung dieser Rechtsregeln hat - faktisch gezwungen, diese nachzuvollziehen. Zu Recht wird daher die Frage aufgeworfen, ob die Terminologie ,autonomer Nachvollzug“ für diese Fallgruppe noch überzeugend ist. ${ }^{65}$

5. Während EU-Richtlinien zum Finanzmarktrecht zumeist auf dem Grundsatz der Mindestharmonisierung basieren und daher dem mitgliedstaatlichen Gesetzgeber nur eine Abweichung nach „oben“ erlaub ist („Gold Plating“), kann der Schweizer Gesetzgeber nach „oben“ un ,,unten“ abweichen (,Swiss Finish“). Will er allerdings einen Marktzu- gang für seine Anbieter erreichen, ist das Ausmaß der Abweichung regelmäßig sehr begrenzt, weil ansonsten die Äquivalenz des Schweizer Rechts mit der einschlägigen EU-Regelung fehlt.

6. Sofern es sich um autonom nachvollzogenes Recht handelt, wendet das Schweizer Bundesgericht - im Rahmen der zu beachtenden nationalen Auslegungsgrundsätze - den Grundsatz der europarechtskonformen Auslegung an. Dies ist überzeugend, denn die mit der Übernahme des EU-Rechts bezweckte Angleichung würde faktisch beseitigt, wenn die nachfolgende Rechtsanwendung sich vom Vorbild entfernt. Dies gilt erst recht, wenn die Angleichung an das EU-Recht den Marktzugang für Schweizer Finanzintermediäre bezweckt, denn dieser Zugang wäre gefährdet, wenn das heimische Recht durch eine die europäischen Vorgaben missachtende Auslegung plötzlich nicht mehr äquivalent wäre.

7. Am Beispiel des autonomen Nachvollzugs lässt sich sehr schön aufzeigen, dass es dem Schweizer Gesetzgeber gelingt, komplexe Schachtelsätze aus EU-Richtlinie in eine klare und verständliche Sprache umzusetzen.

65 Forstmoser (Fn. 13), 531; Heinemann (Fn. 1), 20; Kunz (Fn. 35), 48 